Chronic Obstructive Pulmonary Diseases:

Journal of the COPD Foundation

FOUNDATION

\title{
Original Research \\ Recruiting Patients After Hospital Discharge for Acute Exacerbation of COPD: Challenges and Lessons Learned
}

Gary T. Ferguson, $\mathrm{MD}^{1}$ Bonnie Beck, $\mathrm{PhD}^{2}$ Emmanuelle Clerisme-Beaty, $\mathrm{MD}^{2}$ Dacheng Liu, $\mathrm{PhD}^{2}$ Byron M. Thomashow, MD ${ }^{3}$ Robert A. Wise, MD ${ }^{4}$ Richard ZuWallack, MD ${ }^{5}$ Barry J. Make, MD 6

\section{Abstract}

Background: Hospitalizations for acute exacerbations of chronic obstructive pulmonary disease (AECOPD) are associated with increased mortality and decreased quality of life. Replicate hospital discharge studies were initiated to examine efficacy and safety of once-daily tiotropium HandiHaler ${ }^{\circledast}$ versus placebo, in addition to usual care, in patients discharged from the hospital after an AECOPD.

Methods: Both studies were randomized, placebo-controlled, double-blind, parallel-group, multicenter, with inclusion/exclusion criteria providing a diverse COPD patient cohort hospitalized for $\leq 14$ days with AECOPD. Patients received tiotropium or placebo, initiated within 10 days post-discharge. Target recruitment was 604 patients/study and planned duration was event-driven, ending after 631 clinical outcome events across both studies. Inability to reach targeted site numbers and patient recruitment/retention difficulties led to early study termination. Recruitment/retention challenges and protocol amendment impacts were assessed qualitatively to understand the major issues.

Results: Over 18 months, 219 patients were enrolled; 158 were randomized and 61 failed screening. Premature treatment discontinuation occurred in 49(31\%) patients, of whom 20(41\%) completed health status follow-up. All-cause, 30-day hospital readmission was low (8[5\%] patients). A total of 154(98\%) patients had a concomitant diagnosis and most took pulmonary medication pre-randomization (143[91\%]) and during study treatment (144[92\%]). Inclusion/exclusion criteria changes failed to improve recruitment. Recruitment/retention barriers were identified, relating to patient and clinician factors, health care infrastructure, and clinical practices.

Conclusions: Although AECOPD hospitalization is clinically important and incurs high costs, significant challenges exist in studying this population in clinical trials after hospitalization. Studies are needed to evaluate effective management of AECOPD patients at high risk of adverse clinical outcomes.

\footnotetext{
Abbreviations: acute exacerbation of chronic obstructive pulmonary disease, AECOPD; chronic obstructive pulmonary disease, COPD; Hospital Readmissions Reduction Program, HRRP; Centers for Medicare and Medicaid Services, CMS; institutional review board, IRB; medical science liaison, MSL; adverse event, AE; termination of trial medication, TTM; Medical Dictionary for Regulatory Activities, MedDRA; forced expiratory volume in 1 second, FEV $\mathbf{1}$; forced vital capacity, FVC

Funding Support: This work was supported by Boehringer Ingelheim Pharmaceuticals, Inc.

Date of Acceptance: May 16, 2017

Citation: Ferguson GT, Beck B, Clerisme-Beaty E, et al. Recruiting patients after hospital discharge for acute exacerbation of COPD: Challenges and lessons learned. Chronic Obstr Pulm Dis. 2017;4(4):265-278. doi: https://doi.org/10.15326/jcopdf.4.4.2016.0176
}

\section{This article contains an online supplement.}

1 Pulmonary Research Institute of Southeast Michigan, Farmington Hills, Michigan Boehringer Ingelheim Pharmaceuticals, Inc., Ridgefield,
Connecticut
3 College of Physicians and Surgeons, Columbia University, New York, New York

4 Johns Hopkins University School of Medicine, Baltimore, Maryland 
5 St Francis Hospital Medical Center, Hartford, Connecticut

6 National Jewish Health, Denver, Colorado

\section{Address correspondence to:}

Gary T. Ferguson, MD

Pulmonary Research Institute of Southeast Michigan

29255 West 10 Mile Road, Suite A

Farmington Hills, MI 48336, USA

Phone: 248-478-6561

E-mail: garytferguson@msn.com

\section{Keywords:}

adverse clinical outcomes; AECOPD; clinical trials; recruitment; retention; tiotropium; maintenance therapy

\section{Introduction}

Recurrent episodes of acute exacerbations of chronic obstructive pulmonary disease (AECOPD) requiring hospitalization, have been shown to accelerate lung function decline, increase morbidity and mortality risk, and adversely affect health-related quality of life. ${ }^{1-3}$ Therefore, therapies that reduce the frequency and impact of AECOPD are highly desirable.

Patients with an AECOPD are particularly at high risk of having future exacerbations, ${ }^{4}$ and following an initial COPD-related hospitalization are more likely to have another COPD-related rehospitalization within 1 year. ${ }^{5}$ Around $20 \%$ of patients discharged from the hospital following a COPD-related hospitalization have an all-cause readmission within 30 days of discharge, ${ }^{6-8}$ with the cost of each readmission being around $\$ 12,000$ in 2013 in the United States. ${ }^{7}$ Therefore, in addition to deleterious effects on patients' health and well-being associated with AECOPD, there is a considerable economic burden placed on hospitals. ${ }^{9}$ In the United States in 2012, the Hospital Readmissions Reduction Program (HRRP) was implemented by the Centers for Medicare and Medicaid Services (CMS), with the aim of reducing hospital readmissions in patients who had been hospitalized for acute myocardial infarction, pneumonia, or heart failure. This program was widened in 2014 to include patients hospitalized for AECOPD. ${ }^{10}$ Hospitals now incur financial penalties, in the form of reduced treatment reimbursement, for Medicare patients with unplanned rehospitalizations for COPD within 30 days of discharge. These financial penalties were designed to provide an incentive for hospitals to improve the standard of care they provide to patients and to reduce rehospitalizations. Therefore, COPD- related rehospitalization has become a timely and important issue for health care providers.

Patients with COPD are often undertreated, ${ }^{11}$ and when hospitalized for AECOPD are often discharged on sub-optimal treatment regimens with no provision of maintenance therapy. ${ }^{12,13}$ In several clinical trials, the long-acting anticholinergic bronchodilator, tiotropium, has been shown to reduce the risk and frequency of exacerbations, COPD-related hospitalizations, and patient mortality. ${ }^{14-18}$ With these benefits in mind, twin/replicate hospital discharge studies were designed to investigate the impact of early initiation of maintenance therapy with tiotropium compared with placebo, in addition to usual care, in patients discharged from hospitalization for an AECOPD. Unfortunately, both studies faced problems in reaching target recruitment levels, and the retention of suitable randomized participants was also challenging. Many patients were already receiving protocol-restricted medications for COPD (oral $\beta$-adrenergics or longacting anticholinergics). These difficulties resulted in the studies being terminated early. Furthermore, since the number of randomized patients was very low, the studies had insufficient statistical power to detect clinically meaningful treatment differences.

Nevertheless, information can be gleaned from these trials. Review of methodological issues, the impact of protocol amendments designed to enhance recruitment, and questionnaires submitted to research sites asking questions related to locally observed recruitment and retention obstacles provided information to explore the challenges faced in clinical trial design in studies to follow patients after hospital discharge for an AECOPD. It is anticipated that a better understanding of the issues faced could lead to recommendations that might enable future clinical trials to be successful. In this article, we summarize the study findings and describe the identified barriers to recruitment and retention, relating to patient and clinician factors, health care infrastructure, and current clinical practices.

\section{Methods}

\section{Background}

The twin studies were designed to investigate the impact of early initiation of maintenance therapy with tiotropium compared with placebo, in addition to usual care in patients after a hospitalization for an AECOPD. However, failed study recruitment and 
retention prevented study completion, making primary and secondary endpoint data underpowered for any meaningful analyses. To understand more clearly why these studies were unsuccessful, problems with the original study design were identified, amendments and measures taken in an attempt to remedy the issues are described, along with their ensuing impact on recruitment and retention, and site-specific openended questions related to study challenges were reviewed.

\section{Study Design}

Twin/replicate, randomized, placebo-controlled, double-blind, parallel-group, multicenter, phase IV, hospital discharge studies, conducted in the United States (ClinicalTrials.gov identifiers: NCT01663987 and NCT01662986), aimed to evaluate the safety and efficacy of once-daily maintenance therapy with tiotropium $18 \mu \mathrm{g}$ delivered via HandiHaler ${ }^{\circledR}$ (Boehringer Ingelheim, Ingelheim am Rhein, Germany) compared with placebo in patients after hospital discharge for an AECOPD. Full details of the study design are presented in Table 1 and a schematic of the study design is presented in Figure 1. In addition to receiving tiotropium or placebo, the patients received usual care for COPD as determined by their physician. The planned primary endpoints for the study are detailed in Table 1.

The study was carried out in compliance with the clinical study protocol, and in accordance with the principles of the Declaration of Helsinki, the International Conference on Harmonisation for Good Clinical Practice, and applicable regulatory requirements. The protocol, informed consent, and patient information were reviewed and approved by local institutional review boards (IRB)/independent ethics committees and other regulatory authorities as required by local laws and regulations. The most frequently used IRB by both studies was Schulman Associates IRB, (4445 Lake Forest Drive, Suite 300, Cincinnati, Ohio 45242); a full list of IRBs is provided in the online supplement Table S1. All patients provided written informed consent prior to study participation.

\section{Selection of the Study Population}

The inclusion and exclusion criteria were designed to include a diverse population of patients with COPD, recently hospitalized for an AECOPD, regardless of disease severity. Patients entering screening (Visit 0) had a primary hospital admission diagnosis of AECOPD and a COPD diagnosis, which had to be confirmed by spirometry prior to randomization at Visit 1 . The full patient inclusion and exclusion criteria, as initially set in the protocol, are presented in the online supplement Table S2. Protocol amendments were implemented in an attempt to improve patient recruitment. The key inclusion and exclusion criteria along with their respective amendments are presented in Table 2 .

\section{Patient Recruitment and Retention Issues}

During the recruitment period, barriers to site initiation and patient recruitment were investigated, by means of qualitative data collection. Questionnaires on barriers to recruitment were completed by the majority of sites during in-person visits by medical science liaisons (MSLs). Changes to the study conduct were implemented in an attempt to enhance recruitment.

\section{Statistical Analyses}

The studies were prematurely discontinued due to insufficient patient enrollment and difficulties in identifying sites. Lower than expected patient enrollment and retention in the studies resulted in them being underpowered for statistical analyses; therefore, it was pre-specified that the primary endpoint data analyses (Table 1) would only be descriptive. Data collected via questionnaires on barriers to recruitment were qualitative in nature, and so could not be analyzed statistically.

\section{Safety}

All serious and non-serious adverse events (AEs) occurring during the studies were recorded on study visits and via telephone calls.

\section{Results}

\section{Study Challenges}

\section{Site Initiation}

Over 2000 sites were surveyed for possible study participation, and even after 18 months, it was not possible to initiate the planned number of sites. Over both studies, 100 centers were initiated $(71 \%$ of the target; Figure 2) and of these centers only 54 actively enrolled patients. 


\section{Table 1. Study Design and Primary Endpoints for the Twin Studies}

\begin{tabular}{|c|c|}
\hline Design & $\begin{array}{l}\text { Twin/replicate, randomized, placebo-controlled, double-blind, parallel-group, multicenter, } \\
\text { phase IV, studies }\end{array}$ \\
\hline $\begin{array}{l}\text { Planned Number of } \\
\text { Study Sites and Patients }\end{array}$ & $\begin{array}{l}\text { 50 U.S. sites per study, with a total enrollment per study of } 604 \text { patients (1208 across } \\
\text { both studies) }\end{array}$ \\
\hline Planned Duration & $\begin{array}{l}\text { Event-driven, ending after } 631 \text { patients across both studies experienced an adverse primary } \\
\text { clinical outcome (COPD exacerbation, all-cause re-hospitalization, or all-cause mortality) }\end{array}$ \\
\hline $\begin{array}{l}\text { Planned Recruitment } \\
\text { and Treatment Period }\end{array}$ & $12-18$ months; patients to be on study medication for $1-2$ years \\
\hline Follow-up & $\begin{array}{l}\text { - All patients to remain randomized and followed until study close-out (minimum treatment } \\
\text { period of } 12 \text { weeks) } \\
\text { - End of the study or premature treatment discontinuation, patients to be contacted by a } \\
\text { 30-day follow-up telephone call to collect data on smoking status, exacerbations, adverse } \\
\text { events, concomitant therapies, and health care resource utilization } \\
\text { - Patients discontinuing study medication early to be contacted every } 6 \text { months, after the } \\
\text { 30-day telephone call, until the defined study end, to obtain data on vital status, COPD } \\
\text { exacerbations, concomitant respiratory medication use, and rehospitalizations }\end{array}$ \\
\hline Primary Endpoints & $\begin{array}{l}\text { - Individual studies: Trough } \mathrm{FEV}_{1} \text { at } 12 \text { weeks on study medication } \\
\text { - Trough } \mathrm{FEV}_{1} \text { was defined as the } \mathrm{FEV}_{1} \text { measurement, prior to the next dose of study drug, } \\
\text { and approximately } 24 \text { hours after the last inhalation of study drug } \\
\text { - Combined studies: Time to first adverse clinical outcome event after randomization, which } \\
\text { was a composite endpoint of either COPD exacerbations, or all-cause re-hospitalization, or } \\
\text { all-cause mortality } \\
\text { - COPD exacerbation was defined as a complex of lower respiratory events and symptoms } \\
\text { (either increased or new onset) related to the underlying COPD, with a duration of } \geq 3 \text { days } \\
\text { that required a change in treatment. The complex of lower respiratory events and symptoms } \\
\text { was defined as at least } 2 \text { of the following: shortness of breath, sputum production (volume), } \\
\text { occurrence of purulent sputum, cough, wheezing, chest tightness. A change in treatment } \\
\text { was either the prescription of antibiotics and/or systemic steroids, and/or a newly prescribed } \\
\text { maintenance respiratory medication (i.e., bronchodilators including theophyllines and } \\
\text { phosphodiesterase-4 inhibitors) }\end{array}$ \\
\hline
\end{tabular}

$\mathrm{FEV}_{1}=$ forced expiratory volume in 1 second

\section{Patient Recruitment and Retention}

Patient disposition is shown in Figure 3. The recruitment period was 1 year and 2 months in duration and, of the 219 patients who were screened, $28 \%$ (61 patients) failed the screening process. There were no screen failures recorded due to prior use of tiotropium, this is likely because investigators reviewed medication data prior to approaching a patient with regard to study screening, and if they were already on tiotropium would not have been deemed suitable for screening. The number of participants excluded on this basis is unknown, although reported as a key limitation by investigators. Of the 61 patients who failed screening, 42 patients (69\% of screen failures, 19\% of all participants enrolled) failed to meet spirometry criteria for COPD as defined in the protocol prior to randomization. Only 158 patients were randomized, which was $13 \%$ of the target enrollment (Figure 2).

Across both studies, 49 (31\%) patients prematurely discontinued, of whom 20 (41\%) consented for vital status follow-up. The reasons for premature discontinuation, as listed on the investigator records on the termination of trial medication (TTM) form, are given in Figure 3. According to these records, 18 (37\%) patients discontinued due to AEs. From the specific AE reporting data, according to the Medical Dictionary for 


\section{Figure 1. Study Design}

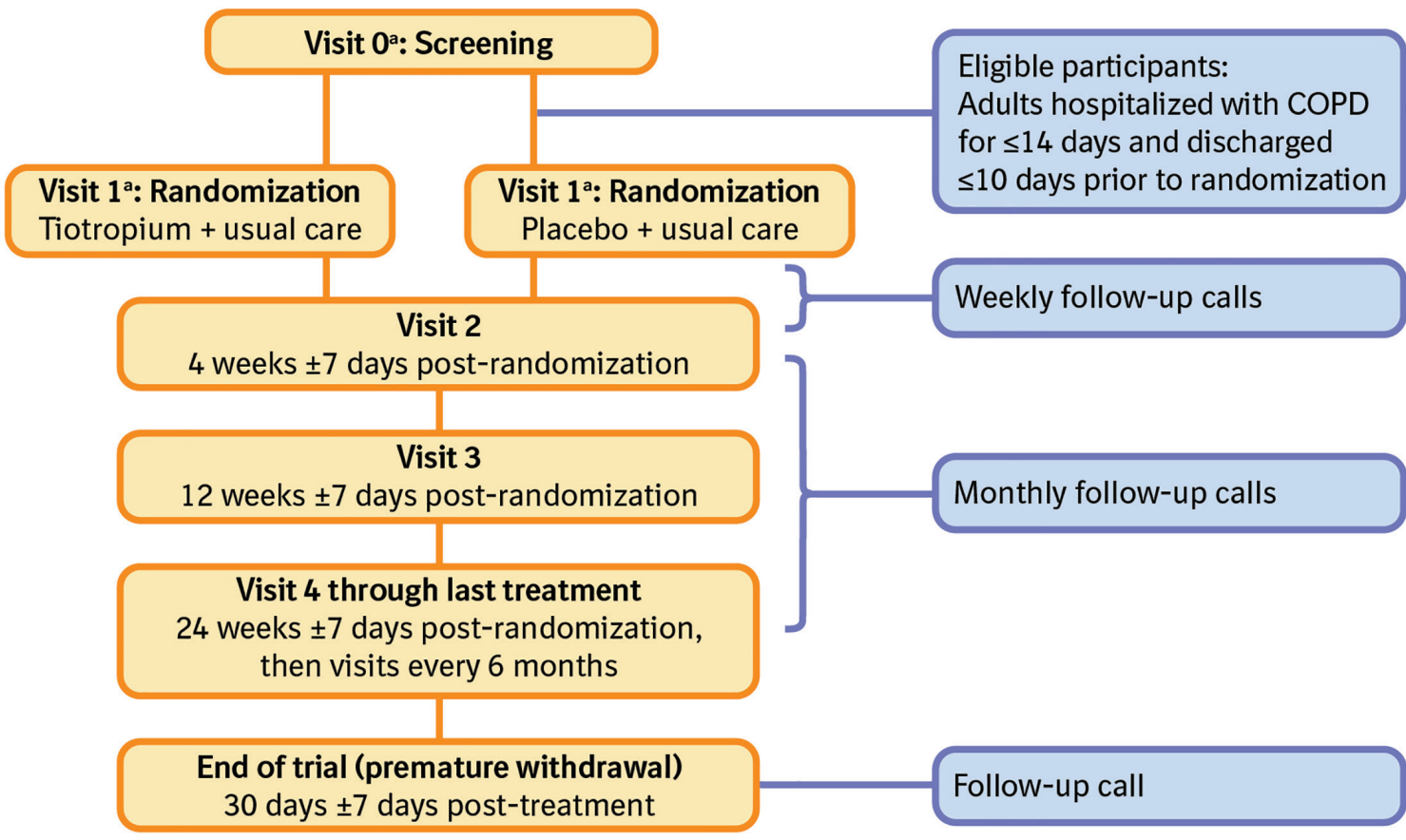

aVisits 0 and 1 could occur on the same day or within 72 hours

Regulatory Activities (MedDRA), of the 14 patients who discontinued, 8 patients discontinued due to the primary system organ class Respiratory, Thoracic, and Mediastinal disorders, with preferred terms of COPD $(n=7)$ and respiratory failure $(n=2) ; 1$ patient had both conditions as well as influenza bronchitis. Other reasons for discontinuation according to the TTM form included: 1 patient due to worsening of other pre-existing disease, 10 patients due to other AEs, and $31(63 \%)$ patients due to administrative reasons (5 patients due to non-compliance with the protocol, 11 patients were lost to follow-up, 9 patients refused to continue with the study medication and 6 patients discontinued for other reasons). The observed allcause, 30-day hospital readmission was low (8 [5.1\%] patients; 4 patients/treatment).

\section{Barriers to Recruitment}

Questionnaires on barriers to recruitment were completed by the majority of sites during in-person visits by MSLs, and the main challenges identified are summarized in Figure 4.

\section{Measures Taken to Improve Enrollment}

\section{Protocol and Procedural Changes}

A variety of changes over the course of the study were implemented in an attempt to enhance patient recruitment and to make recruitment more inclusive. The patient enrollment period was extended from 1 year to up to 18 months, and the number of target sites increased from 50 to 70 centers per study. As detailed in Table 1, several inclusion and exclusion criteria were revised by protocol amendment, to make them more reflective of the general population and to make them more inclusive for patients. The requirement for visits to occur between 7 am and 10 am was removed, so that visits could be more accommodating for patients. Initially, screen-failed patients were not allowed to reenter the study; this restriction was lifted, allowing patients who screen failed and subsequently had another COPD exacerbation requiring hospitalization to be re-evaluated for study eligibility. Despite these measures, the protocol amendments do not appear to have substantially improved enrollment as shown in Figures 2 and 5. 


\section{Table 2. Original Selected Key Inclusion and Exclusion Criteria, and Protocol Changes Initiated to Improve Patient Recruitment}

\section{Original Criteria \\ Protocol Amendments to Improve Patient Recruitment}

Inclusion Criteria: Visit 0

Signed informed consent

Males/females, aged $\geq 40$ years

Primary diagnosis of AECOPD at hospital admission and

hospital length of stay $\leq 14$ days

Inclusion Criteria: Visit 1

Patient discharged from hospital $\leq 10$ days from

randomization

Clinical diagnosis of COPD and documented airway

obstruction with post-bronchodilator $\mathrm{FEV}_{1} \leq 80 \%$

predicted $^{\mathrm{a}}$ and $\mathrm{FEV}_{1} / \mathrm{FVC}<0.7$ (spirometry performed at

Visit 1 or prior spirometric evidence)

Current or ex-smoker with smoking history of $\geq 10$ pack

years

\section{Exclusion Criteria: Visit 0}

Therapy with any long-acting inhaled anticholinergic $\leq 14$

days prior to hospitalization; or any other restricted concomitant medications

$>30$ days of long-acting inhaled anticholinergic use within the 3 months prior to hospital discharge or in the period between hospital discharge and randomization; or any other restricted concomitant medications

\section{Exclusion Criteria: Visit 1}

Recent history ( $\leq 6$ months) of myocardial infarction

History of myocardial infarction during the hospitalization preceding randomization

Any unstable or life-threatening cardiac arrhythmia requiring intervention or change in drug therapy during

the last year

History of thoracotomy with pulmonary resection Hospitalization for cardiac failure $\leq 1$ year

Significant symptomatic prostatic hyperplasia or bladder-neck obstruction (patients with controlled symptoms may be included)

Treatment with any restricted pulmonary medication (oral $\beta$-adrenergics, long-acting anticholinergics, investigational drugs)

Residence in a nursing home assisted living facility

$\mathrm{FEV}_{1}=$ forced expiratory volume in 1 second; $\mathrm{FVC}=$ forced vital capacity

${ }^{\mathrm{a}} \mathrm{FEV}_{1} \leq 80 \%$ predicted criterion was added at the time of protocol amendment to better ensure patients fulfilled a diagnosis of COPD

\section{Other Initiatives}

Site-specific recruitment plans were developed by a patient recruitment specialist, and included such initiatives as local advertisement of the

\section{Criterion deleted}

Hospitalization for cardiac failure in the hospitalization preceding randomization
Discharge to short-term rehabilitation facility or other short-term care facility was permitted, if no plans for long-term nursing home care 


\section{Figure 2. Actual Versus Target Site Initiations and Patient Recruitment}
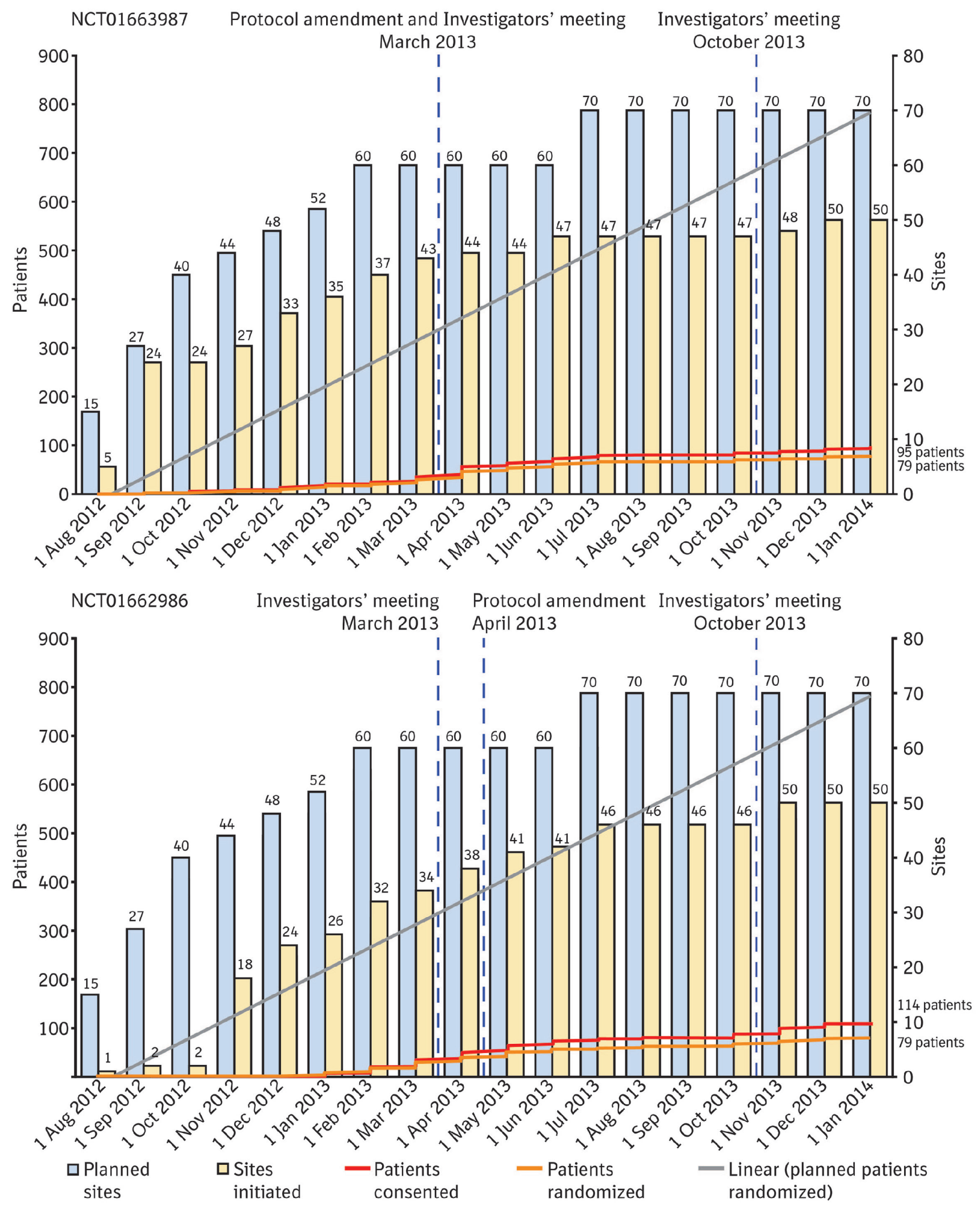


\section{Figure 3. Patient Disposition}

$61 / 219(28 \%)$ patients failed screening:

- 49 inclusion/exclusion criteria

-42 pulmonary function

- 5 consent withdrawn (not due

to adverse event)

- 1 adverse event

- 5 other

- 1 reason unknown

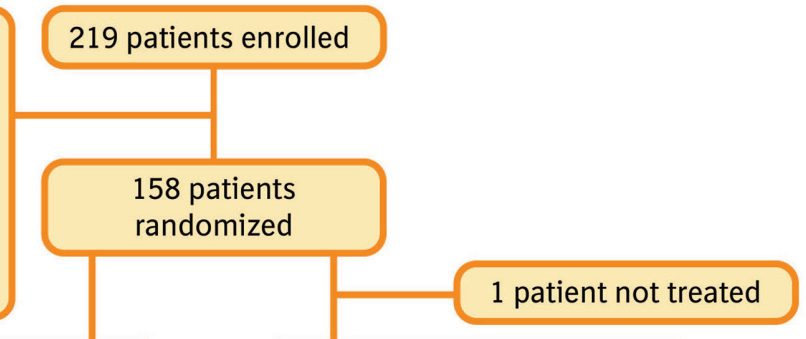

78 patients received placebo

$30 / 78(38 \%)$ patients discontinued:

12 adverse events:

5 worsening of study disease

-7 other adverse event

18 administrative reasons:

2 non-compliant with protocol

6 lost to follow-up

4 refused to continue medication 6 other reason

$15 / 30(50 \%)$ patients had vital-status follow-up

(26\%) patients ha

vital-status follow-up

60/79 (76\%) completed

tiotropium treatment
48/78 (62\%) completed placebo treatment

Data Source: Termination of Trial Medication Form Investigator Records

\section{Figure 4. Patient Recruitment Challenges}

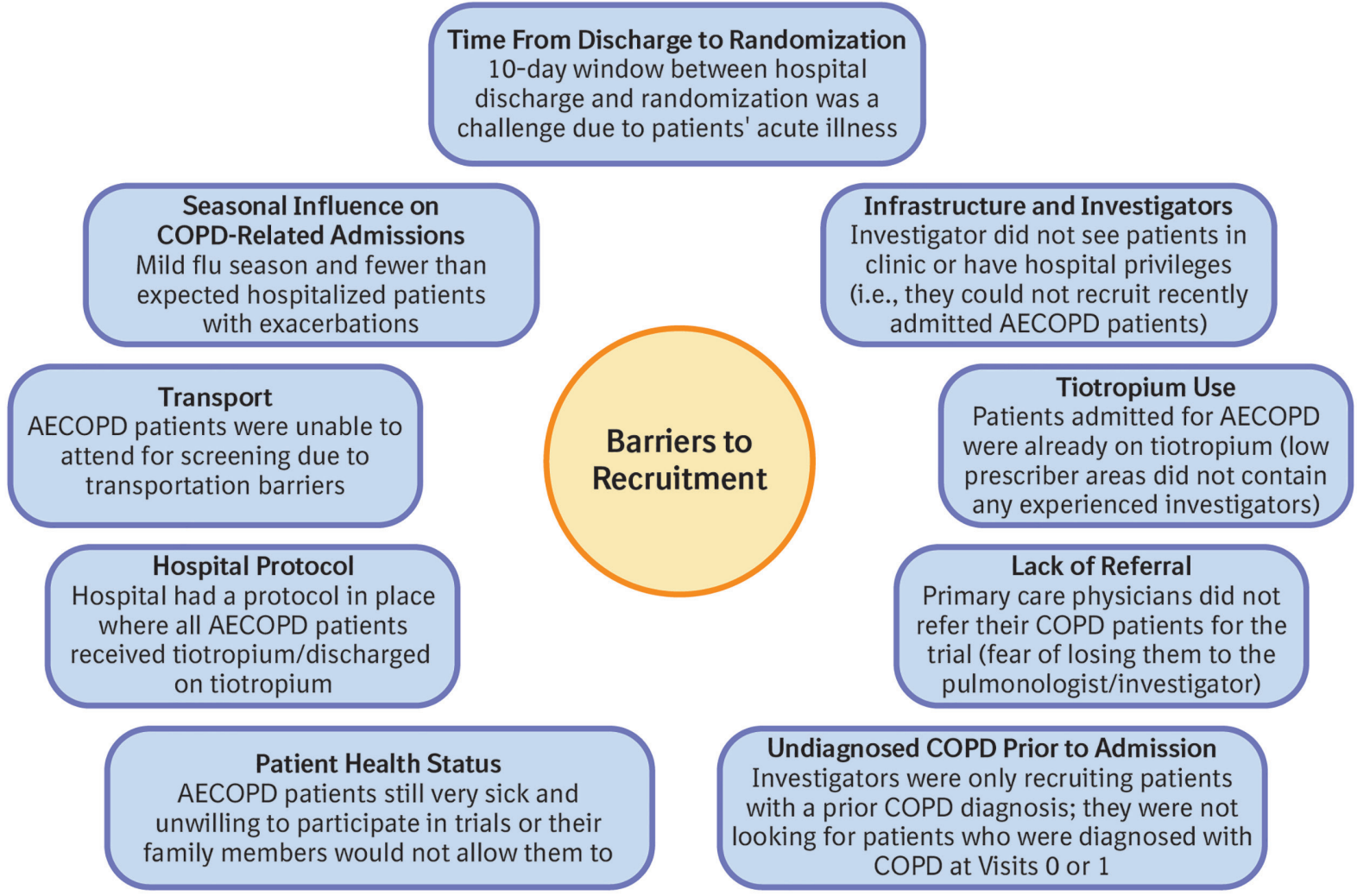


Figure 5. Patient Recruitment Rate per Site per Month
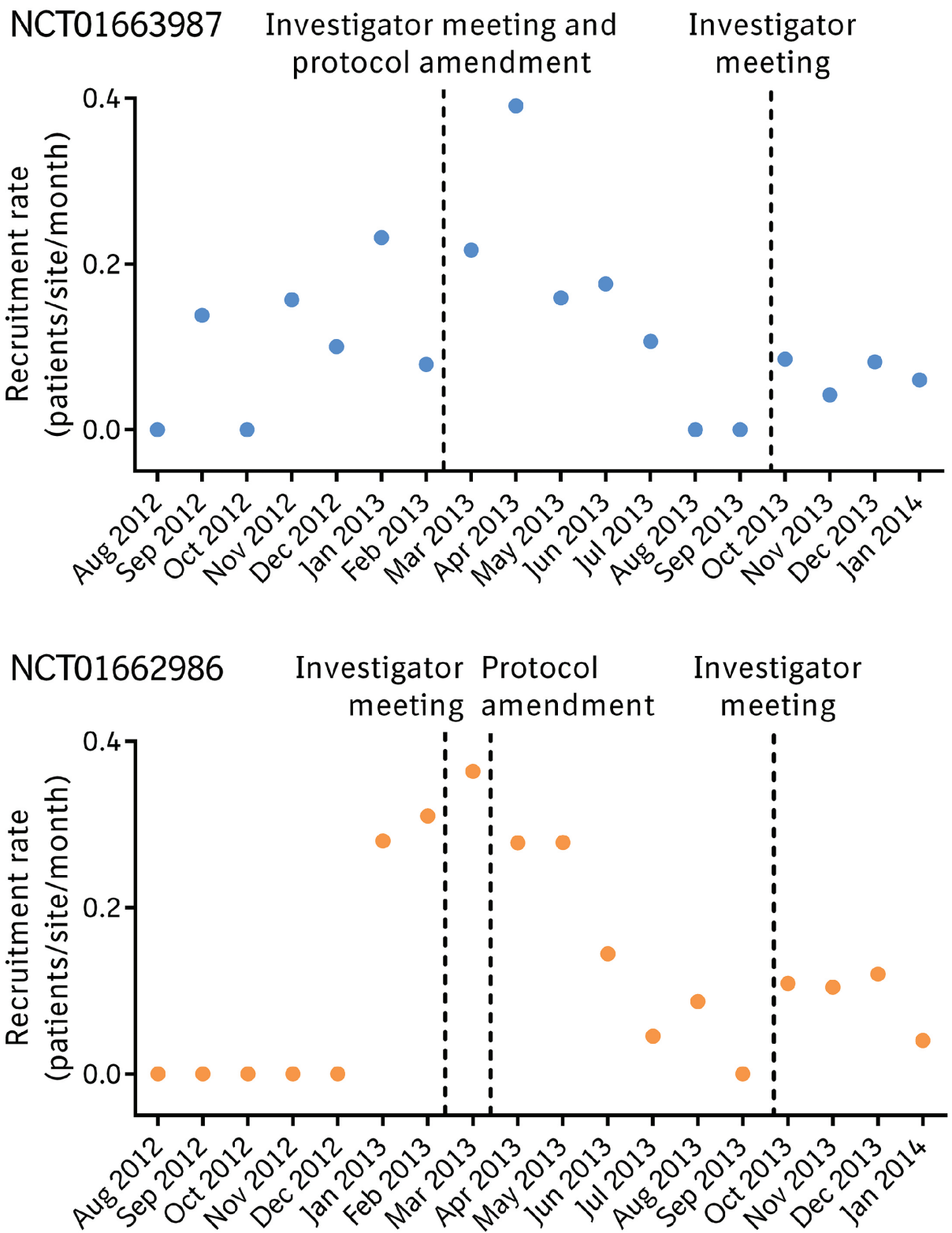

Assessment of recruitment was mostly conducted towards the end of each month.

site-specific recruitment plans were reviewed and regular enrollment and recruitment updates were made. A recruitment pocket guide was given to the investigators, which detailed the study endpoints and key inclusion and exclusion criteria. Point-of-care tools were developed by the study team to assist with recruitment, retention, and participant support and these were provided to the sites. The point-of-care tools included: a patient welcome folder and letter, a study description leaflet for patients, a visit-by-visit planner, an AE sheet, visit "thank you" cards, and tote bags.

Several initiatives were put in place to raise awareness of the studies for potential sites, clinicians, and prospective patients. Posters were made available at study sites. An article was published in the COPD Foundation's Lung Health Professional magazine ${ }^{19}$ detailing the aims of the studies and providing contact details for patient referral; reprints of the article were made available by the COPD Foundation at their booth at the American Thoracic Society International 
Conference in Philadelphia, May 17-22, 2013.

Investigators' meetings were held, at which discussions took place on recruitment and retention strategies. Pre-hospitalization strategies focused on early activities around education and engagement, for example, the use of hospital analytics to improve patient identification, the creation of a fully aware medical community, a proactive patient outreach before exacerbation, and customized support based on individual site needs. In-hospitalization strategies included identifying potential patients while in the hospital and directly after discharge by creating and optimizing referral networks, monitoring admissions and progress of potential study patients, and identifying specific barriers to recruitment. Posthospitalization measures focused on assisting patients to remain in the studies, by means of logistical, educational, and motivational strategies.

Despite attempts during investigators' meetings to implement measures to increase patient recruitment and retention, enrollment did not improve (Figures 2 and 5).

\section{Study Findings}

Patients' baseline demographic and clinical characteristics, pulmonary medication use, exploratory efficacy results, and safety data are provided in the online supplement as additional text, Tables S3-8, and Figure S1. At baseline, most patients had moderate-tosevere COPD, and their pre-randomization health care use indicated that they were at high risk for adverse clinical outcomes. Patients' concomitant diagnoses were as expected for a COPD population and most patients were taking pulmonary medication prerandomization and during the study. Lung function data suggested a benefit of tiotropium treatment over placebo, but this was not statistically analyzed due to the small sample size. Interpretation of a Kaplan-Meier curve for time to first adverse clinical outcome event was limited, particularly at the later timepoints, by a decreasing number of at-risk patients, and may have been influenced by the higher discontinuation rate in the placebo group compared with the tiotropium group. The proportions of patients with adverse clinical outcomes were similar for the tiotropium and placebo groups. Based on the observed number of events per patient-year, both the COPD exacerbation and all-cause hospitalization rates were numerically lower for the tiotropium group compared with the placebo group; but again, this could not be statistically analyzed. Adverse events were similar between treatments and were consistent with the known safety profile of tiotropium. ${ }^{20}$

\section{Discussion}

The twin, hospital discharge studies were designed to evaluate the effectiveness of initiating once-daily tiotropium $18 \mu \mathrm{g}$ therapy on patients discharged from the hospital within 10 days of an AECOPD. In addition to receiving tiotropium or placebo, patients received usual care for COPD, as determined by their physician. It was hypothesized that tiotropium maintenance therapy would reduce the frequency and severity of adverse clinical outcomes and the risk of rehospitalization for AECOPD.

Although 30-day readmission is a critical time point relative to the CMS HRRP compensation methods, ${ }^{10}$ readmissions and failed care after hospitalization remain critically important to patients for a much longer period of time beyond discharge. For this study, the length of patient follow-up was chosen to try to better assess the impact on patients, beyond the financial impact on hospitals associated with the 30day readmission policy.

Unfortunately, patient recruitment was much lower than the projected target for both studies, despite multiple attempts to increase enrollment. Underrecruitment resulted in the decision to terminate the studies early, and the data collected were insufficient for clinically meaningful statistical analyses. Over the course of the studies, several barriers to recruitment were identified, which highlighted the challenges faced when performing these types of studies in this specific population, which is at high risk of adverse clinical outcomes.

Although the number of screened patients was slightly higher in one study compared with the other study, the number of patients randomized was similar. These discrepancies between studies are often seen with replicate trials and are not considered significant. Although there were a couple of individual sites more successful at recruiting, no specific differences could be identified for them compared with the other sites.

It has been estimated that less than $50 \%$ of clinical trials meet their recruitment targets without needing an extension to the enrollment period; ${ }^{21}$ however, difficulties in recruitment are rarely published. In 
this hospital discharge study, patients were at high risk of future adverse clinical outcomes, making for a vulnerable patient population. In a study by Ohar et $\mathrm{al}^{22}$ in a similar patient cohort to that in our hospital discharge studies, challenges were faced in recruiting patients to a trial, post discharge from the hospital, an emergency room, or an office visit, following a COPD exacerbation. The recruitment difficulties highlighted included: exclusion of patients due to their high comorbidity burden; difficulties encountered in coordinating a patient's hospitalization, discharge, and study participation consent; and a limited number of investigators who had both outpatient and inpatient practices, qualifying them for study participation. These findings are similar to those encountered in our twin studies. Treweek et $a^{21}$ suggested that trialists, with support from their funders, should include evaluations of recruitment strategies in their clinical trials as this would allow the most effective interventions to be identified for future studies.

Despite findings in the literature, which suggest that $66 \%$ of commercially insured patients and $71 \%$ of Medicare patients with COPD do not receive COPD maintenance therapy, and $59 \%$ and $66 \%$, respectively, have no COPD medication prescriptions, ${ }^{11}$ the populations in our studies were very different, in that only around $10 \%$ of patients were not taking pulmonary medications at baseline. The overall study cohort was also different, in that the all-cause, 30-day hospital readmission rate was strikingly low: $5 \%$ compared with other readmission estimates of around 20\%. ${ }^{6-8}$ The reason for this discrepancy is not clear, but it may be biased by the fact that patients were followed closely as part of the protocol, which may not reflect clinical practice. Thus, the studies were terminated early, not only because of their low recruitment levels, but also because the patient cohort enrolled in the studies seem to be quite different to that expected from the literature describing real-world COPD populations. Readmission rates may be reflective of the low followup rates achieved during the studies.

Of the participants admitted to the hospital with a diagnosis of AECOPD, 19\% of those screened did not meet the spirometric criteria for COPD and were excluded; this discrepancy between diagnosis of AECOPD at admission and subsequent spirometry has also been noted in previous studies. ${ }^{23,24}$ Furthermore, in our studies, the premature discontinuation of study treatment was high (31\%), with only $41 \%$ of those who dropped out of the studies having vital status follow up; this made it difficult to determine long-term health status. The follow-up frequency of every 6 months may have been too infrequent, and planned visits rather than telephone calls may have been a more effective form of follow-up.

\section{Conclusions}

It will be very difficult to design future drug trials for a vulnerable COPD population (with high risk of adverse clinical outcomes) that are ethically acceptable if they include placebo or under-treated control groups and extend for a relatively long duration. Thus, investigations on novel therapies may require testing the safety and efficacy of "add on" therapies. We present our experience as suggested by Treweek et $\mathrm{al}^{21}$ to allow the most effective study design and recruitment strategy interventions to be identified for future investigations in similar patient groups.

\section{Acknowledgments}

Writing, editorial support, and formatting assistance was provided by Jane M. Gilbert BSc, CMPP of Envision Scientific Solutions, which was contracted and funded by Boehringer Ingelheim Pharmaceuticals, Inc., for these services.

\section{Declaration of Interest}

The authors received no direct compensation related to the development of the manuscript. Boehringer Ingelheim Pharmaceuticals, Inc., was given the opportunity to review the manuscript for medical and scientific accuracy as well as intellectual property considerations.

Gary T. Ferguson reports personal fees, grants, and non-financial support from Boehringer Ingelheim during the conduct of the study. Outside the submitted work, he reports non-financial support from Boehringer Ingelheim; personal fees from AstraZeneca, Boehringer Ingelheim, Forest, GlaxoSmithKline, Innoviva, Meda, Mylan, Novartis, Pearl Therapeutics, Sunovion, and Verona; and grants from Aquinox, AstraZeneca, Boehringer Ingelheim, Forest, Novartis, Pearl Therapeutics, Sunovion, and Theravance. Byron M. Thomashow reports personal fees from Boehringer Ingelheim during the conduct of the study; and personal fees from AstraZeneca, Boehringer Ingelheim, Forrest, GlaxoSmithKline, 
InterMune, and Novartis outside the submitted work. Robert A. Wise reports personal fees and grants from Boehringer Ingelheim during the conduct of the study. He reports personal fees from AstraZeneca, Boehringer Ingelheim, ContraFect, GlaxoSmithKline, Janssen, Merck, Novartis, Pfizer, Pulmonx, Roche, Spiration, Sunovion, Teva, Theravance, Verona, and Vertex; and grants from AstraZeneca, Boehringer Ingelheim, GlaxoSmithKline, and Pearl Therapeutics outside the submitted work. Richard ZuWallack's institution has received research grants, consulting fees, and honoraria from Boehringer Ingelheim Pharmaceuticals, and honoraria from AstraZeneca and GlaxoSmithKline. Barry J. Make reports fees for medical advisory boards and research studies from Boehringer Ingelheim during the conduct of the study. He reports personal fees from AstraZeneca, Boehringer Ingelheim,
CSL Behring, Forest, GlaxoSmithKline, Novartis, Sunovion, and Verona; grants from AstraZeneca, Boehringer Ingelheim, GlaxoSmithKline, Pearl, and Sunovion; funds for CME from American College of Chest Physicians, Cleveland Clinic, Consensus Medical Education, Foundation for Improving Patient Outcomes, Hybrid Communications, Integrity Medical Education, Medscape, Mount Sinai Medical Center, National Jewish Health, Peer Review Institute, Projects in Knowledge, Spiration, SPIRE Learning, and WebMD; Data Safety Monitoring Board fees from Spiration; and royalties from Up-To-Date outside the submitted work. Bonnie Beck, Emmanuelle Clerisme-Beaty, and Dacheng Liu are employees of Boehringer Ingelheim Pharmaceuticals, Inc. 


\section{References}

1. Johannesdottir SA, Christiansen CF, Johansen MB, et al. Hospitalization with acute exacerbation of chronic obstructive pulmonary disease and associated health resource utilization: a population-based Danish cohort study. J Med Econ. 2013; 16(7): 897-906. doi: https://doi.org/10.3111/13696998.2013.800525

2. Wang Q, Bourbeau J. Outcomes and health-related quality of life following hospitalization for an acute exacerbation of COPD. Respirology. 2005; 10(3): 334-340.

doi: https://doi.org/10.1111/j.1440-1843.2005.00718.x

3. Piquet J, Chavaillon JM, David P, et al. High-risk patients following hospitalisation for an acute exacerbation of COPD. Eur Respir J. 2013; 42(4): 946-955.

doi: https://doi.org/10.1183/09031936.00180312

4. Müllerová H, Shukla A, Hawkins A, Quint J. Risk factors for acute exacerbations of COPD in a primary care population: a retrospective observational cohort study. BMJ Open. 2014; 4(12): e006171. doi: https://doi.org/10.1136/bmjopen-2014-006171

5. Baker CL, Zou KH, Su J. Risk assessment of readmissions following an initial COPD-related hospitalization. Int J Chron Obstruct Pulmon Dis. 2013; 8: 551-559. doi: https://doi.org/10.2147/COPD.S51507

6. Jencks SF, Williams MV, Coleman EA. Rehospitalizations among patients in the Medicare fee-for-service program. $N$ Engl J Med. 2009; 360(14): 1418-1428.

doi: https://doi.org/10.1056/NEJMsa0803563

7. Agency for Healthcare Research and Quality. Healthcare Cost and Utilization Project: 2013 Hospital Readmissions. HCUPnet website. http://hcupnet.ahrq.gov. Published 2013. Accessed October 19, 2016.

8. Shah T, Churpek MM, Coca Perraillon M, Konetzka RT. Understanding why patients with COPD get readmitted: a large national study to delineate the Medicare population for the readmissions penalty expansion. Chest. 2015; 147(5): 1219-1226. doi: https://doi.org/10.1378/chest.14-2181

9. Hasegawa K, Tsugawa Y, Tsai CL, Brown DF, Camargo CA, Jr. Frequent utilization of the emergency department for acute exacerbation of chronic obstructive pulmonary disease. Respir Res. 2014; 15: 40. doi: https://doi.org/10.1186/1465-9921-15-40

10. Centers for Medicare \& Medicaid Services (CMS). Readmissions Reduction Program. CMS website. https:// wWw.cms.gov/medicare/medicare-fee-for-service-payment/ acuteinpatientpps/readmissions-reduction-program.html. Published 2012. Updated April 4, 2016. Accessed February 6, 2017.

11. Make B, Dutro MP, Paulose-Ram R, Marton JP, Mapel DW. Undertreatment of COPD: a retrospective analysis of US managed care and Medicare patients. Int J Chron Obstruct Pulmon Dis. 2012; 7: 1-9.

doi: https://doi.org/10.2147/COPD.S27032
12. Pradan L, Ferreira I, Postolache P. The quality of medical care during an acute exacerbations of chronic obstructive pulmonary disease. Rev Med Chir Soc Med Nat Iasi. 2013; 117(4): 870874.

13. Yip NH, Yuen G, Lazar EJ, et al. Analysis of hospitalizations for COPD exacerbation: opportunities for improving care. COPD. 2010; 7(2): 85-92.

doi: https://doi.org/10.3109/15412551003631683

14. Dusser D, Bravo ML, Iacono P. The effect of tiotropium on exacerbations and airflow in patients with COPD. Eur Respir J. 2006; 27(3): 547-555.

doi: https://doi.org/10.1183/09031936.06.00062705

15. Tashkin DP, Celli B, Senn S, et al. A 4-year trial of tiotropium in chronic obstructive pulmonary disease. $N$ Engl J Med. 2008; 359(15): 1543-1554.

doi: https://doi.org/10.1056/NEJMoa0805800

16. Vogelmeier C, Hederer B, Glaab T, et al. Tiotropium versus salmeterol for the prevention of exacerbations of COPD. $N$ Engl J Med. 2011; 364(12): 1093-1103.

doi: https://doi.org/10.1056/NEJMoa1008378

17. Celli B, Decramer M, Kesten S, et al. Mortality in the 4-year trial of tiotropium (UPLIFT) in patients with chronic obstructive pulmonary disease. Am J Respir Crit Care Med. 2009; 180(10): 948-955. doi: http://dx.doi.org/10.1164/rccm.200906-08760C

18. Bateman ED, Tashkin D, Siafakas N, et al. A one-year trial of tiotropium Respimat plus usual therapy in COPD patients. Respir Med. 2010; 104(10): 1460-1472. doi: https://doi.org/10.1016/j.rmed.2010.06.004

19. Ferguson G, Make B. Effect of tiotropium on patients recovering from an acute exacerbation: study overview. Lung Health Professional. 2013; 4(1): 35-36.

20. Boehringer Ingelheim International GmbH. Label information for Spiriva ${ }^{\circledR}$ HandiHaler $^{\circledR}$ (tiotropium bromide) inhalation powder, for oral inhalation use. Food and Drug Administration website. http://www.accessdata.fda.gov/drugsatfda_docs/label/ 2015/021395s040lbl.pdf. Updated December 2015. Accessed October 19, 2016.

21. Treweek S, Pitkethly M, Cook J, et al. Strategies to improve recruitment to randomised controlled trials. Cochrane Database Syst Rev. 2010; (4): MR000013.

doi: https://doi.org/10.1002/14651858.MR000013.pub5

22. Ohar JA, Crater GD, Emmett A, et al. Fluticasone propionate/

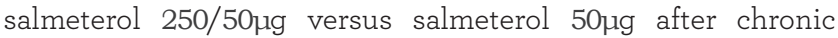
obstructive pulmonary disease exacerbation. Respir Res. 2014; 15: 105. doi: https://doi.org/10.1186/s12931-014-0105-2

23. Stein BD, Bautista A, Schumock GT, et al. The validity of International Classification of Diseases, Ninth Revision, Clinical Modification diagnosis codes for identifying patients hospitalized for COPD exacerbations. Chest. 2012; 141(1): 87-93. doi: https://doi.org/10.1378/chest.11-0024 
24. Prieto-Centurion V, Rolle AJ, Au DH, et al. Multicenter study comparing case definitions used to identify patients with chronic obstructive pulmonary disease. Am J Respir Crit Care Med. 2014; 190(9): 989-995.

doi: https://doi.org/10.1164/rccm.201406-1166OC 